\title{
Effects of Age and Sex on the Single-Dose Pharmacokinetics and Pharmacodynamics of Apixaban
}

\author{
Charles E. Frost • Yan Song • Andrew Shenker • \\ Jessie Wang • Yu Chen Barrett · Alan Schuster • \\ Stuart I. Harris · Frank LaCreta
}

Published online: 9 January 2015

(c) The Author(s) 2015. This article is published with open access at Springerlink.com

\begin{abstract}
Background and Objectives The effects of age and sex on apixaban pharmacokinetics and pharmacodynamics were studied.

Methods This was an open-label, single-dose, $2 \times 2$ factorial study. Healthy young (aged 18-40 years) and elderly (aged $\geq 65$ years) male and female subjects received a single oral $20 \mathrm{mg}$ dose of apixaban. Blood and urine samples were collected for pharmacokinetic and pharmacodynamic (blood only) analyses. Subjects were monitored for adverse events throughout the study.

Results Seventy-nine subjects were enrolled into four groups: young males $(n=20)$, elderly males $(n=20)$, young females $(n=20)$ and elderly females $(n=19)$. Age did not affect the maximum observed plasma concentration
\end{abstract}

C. E. Frost $(\bowtie) \cdot$ Y. Song · A. Shenker ·

Y. C. Barrett - F. LaCreta

Discovery Medicine and Clinical Pharmacology, Bristol-Myers Squibb Research and Development, Mail Stop E12-16, Route 206 and Province Line Road, Princeton, NJ 08543-4000, USA e-mail: charles.frost@bms.com

J. Wang

Global Regulatory, Safety \& Biometrics, Bristol-Myers Squibb Research and Development, Mail Stop E12-16, Route 206 and Province Line Road, Princeton, NJ 08543-4000, USA

\section{A. Schuster}

Bioanalytical Sciences, Bristol-Myers Squibb Company,

Princeton, NJ, USA

S. I. Harris

Seaview Research, Miami, FL, USA
$\left(C_{\max }\right)$. The mean area under the concentration-time curve from time zero extrapolated to infinite time $\left(\mathrm{AUC}_{\infty}\right)$ was $32 \%$ greater in elderly subjects than in young subjects. The mean $C_{\max }$ and $\mathrm{AUC}_{\infty}$ values were 18 and $15 \%$ higher, respectively, in females than in males. The time course of the mean international normalized ratio (INR), modified prothrombin time (mPT) and anti-Xa activity tracked the apixaban concentration-time curve. All three pharmacodynamic measures exhibited a positive linear correlation with the plasma apixaban concentration. Differences in the mean INR, mPT and anti-Xa activity between age and sex groups were small $(<15 \%$ at the maximum mean values) and were generally related to pharmacokinetic differences. However, anti-Xa activity demonstrated less variability than the INR or mPT, and may have utility as a bioassay for apixaban. Apixaban was well tolerated, with no serious adverse events.

Conclusion There were no clinically meaningful age- or sex-related differences in the pharmacokinetics and pharmacodynamics of apixaban that would require dose modification on the basis of age or sex alone.

\section{Key Points}

In this study, there were no clinically meaningful age- or sex-related differences in the pharmacokinetics and pharmacodynamics of apixaban.

Apixaban is safe and well tolerated in both elderly and young subjects of both sexes.

Dose adjustment of apixaban is not required on the basis of age or sex alone. 


\section{Background}

Anticoagulation plays an important role in the prevention and treatment of venous and arterial thrombosis [1-3]. Conventional anticoagulants, such as unfractionated heparin, low molecular weight heparin (LMWH) and warfarin, have limitations that may contribute to suboptimal patient management [3-5]. In recent years, considerable effort has been directed towards the development of orally administered anticoagulants that have a rapid onset of action, predictable pharmacokinetics and pharmacodynamics, and a low propensity for food and drug interactions. Targets of these new oral anticoagulants include clotting factors such as factor $\mathrm{Xa}(\mathrm{FXa})$ and thrombin.

Apixaban is a novel oral, selective, direct-acting, reversible FXa inhibitor, which was approved in several countries for prevention of venous thromboembolism (VTE) in patients following elective knee or hip replacement surgery and for reduction in the risk of stroke or systemic embolism in patients with nonvalvular atrial fibrillation (NVAF), and recently for the treatment of venous thromboembolism. Apixaban has oral bioavailability of $\sim 50 \%$ and multiple pathways of elimination (including renal elimination, metabolism, biliary excretion and direct intestinal excretion), with renal clearance $\left(\mathrm{CL}_{\mathrm{R}}\right)$ accounting for $27 \%$ of total clearance [6-8]. The average elimination half-life $\left(t_{1 / 2}\right)$ is approximately $12 \mathrm{~h}$ (range $8-15 \mathrm{~h}$ ) [9].

Numerous studies have shown that age and sex have the potential to influence the pharmacokinetics and pharmacodynamics of some drugs, including anticoagulants [10-14]. Physiological changes associated with aging, such as increased gastric $\mathrm{pH}$, reduced lean body mass, decreased liver size and hepatic blood flow, and a decreased glomerular filtration rate, can affect the absorption, distribution, metabolism and excretion of drugs [10]. The incidence and prevalence of many thromboembolic disorders, such as NVAF, increase with advancing age, as does the need for major orthopaedic surgery $[1,15]$. Sex-related differences in drug pharmacokinetics have also been attributed to physiological differences between males and females [11, 14]. The primary objective of this study was to assess the effects of age and sex on the single-dose pharmacokinetics of apixaban. Secondary objectives were to assess the effects of age and sex on the single-dose pharmacodynamics of apixaban, along with the safety and tolerability profile.

\section{Methods}

\subsection{Study Design}

This was an open-label, single-dose, $2 \times 2$ factorial study in both young and elderly healthy subjects, who received a single oral $20 \mathrm{mg}$ dose of apixaban (clinical supply; Bristol-Myers Squibb, New Brunswick, NJ, USA). This was the highest dose evaluated in phase 2 studies $[16,17]$ and was expected to be well tolerated on the basis of previous experience [7]. This study was conducted at a single centre (Seaview Research, Miami, FL, USA), and the protocol and informed consent were reviewed and approved by an independent investigational review board (Plantation, FL, USA). The study was conducted in accordance with the codes and guidelines of the Declaration of Helsinki, Good Clinical Practice and all applicable laws and regulations.

Subjects were grouped by age (young and elderly) and sex. Given a null hypothesis of no age- or sex-specific differences in pharmacokinetics, data from 76 subjects (19 subjects per age/sex group) would provide $97 \%$ power to conclude absence of effects with respect to the area under the plasma concentration-time curve (AUC) from time zero extrapolated to infinite time $\left(\mathrm{AUC}_{\infty}\right)$ and the maximum observed plasma concentration $\left(C_{\max }\right)$ [i.e. with the $90 \%$ confidence intervals (CIs) of the geometric mean ratios being within $80-125 \%$ ]. These calculations assumed that there is no age-by-sex interaction and that the $\mathrm{AUC}_{\infty}$ and $C_{\max }$ are log-normally distributed with coefficients of variation of $25 \%$, as estimated from a previous study [7]. To allow for possible dropouts, it was planned that 80 subjects would be treated (20 subjects per age/sex group).

Subjects who signed the informed consent form were enrolled into the following four groups: young men (aged 18-40 years), elderly men (aged $\geq 65$ years), young women (aged 18-40 years) and elderly women (aged $\geq 65$ years). Subjects were admitted to the clinical facility 2 days prior to dosing (day -2 ) and remained until $96 \mathrm{~h}$ postdose (day 5). On day 1 , after fasting overnight for at least $10 \mathrm{~h}$, subjects received a single oral dose of $20 \mathrm{mg}$ apixaban. Blood and urine samples were collected for pharmacokinetic and pharmacodynamic (blood only) analyses at intervals up to $96 \mathrm{~h}$ postdose. Physical examinations (including vital sign measurements), 12-lead electrocardiograms and clinical laboratory evaluations were performed at baseline and prior to study discharge.

\subsection{Study Population}

Healthy male and female subjects aged 18-40 years or $\geq 65$ years and with a body mass index (BMI) of $18-32 \mathrm{~kg} / \mathrm{m}^{2}$ were eligible for the study. The exclusion criteria included evidence or history of a bleeding disorder; history of gastrointestinal disease or surgery that could interfere with the absorption of apixaban; and use of any hormonal contraceptive within 3 months before dosing, any prescription drug or over-the-counter acid controller within 4 weeks before dosing, drugs known to increase the potential for bleeding [such as aspirin, nonsteroidal anti- 
inflammatory drugs (NSAIDs) or anticoagulants] within 2 weeks before dosing or any other drugs (including overthe-counter medications or dietary supplements) within 1 week before dosing.

\subsection{Sample Collection and Assay}

Venous blood samples $(4.5 \mathrm{~mL})$ were collected in sodium citrate $(3.2 \%)$ tubes at $0 \mathrm{~h}$ (predose) and at $1,1.5,2,3,4$, $6,8,10,12,18,24,36,48,72$ and $96 \mathrm{~h}$ postdose for the pharmacokinetic and pharmacodynamic analyses. Pharmacokinetic blood samples were centrifuged for $15 \mathrm{~min}$ at $1,500 \times g$ at $4{ }^{\circ} \mathrm{C}$, and the plasma was transferred to $4 \mathrm{~mL}$ cryogenic vials, which were frozen immediately in dry ice and stored for shipment at $-20{ }^{\circ} \mathrm{C}$. Following a predose void, subjects provided urine samples for the time intervals of 0-12, 12-24, 24-36, 36-48, 48-60, 60-72 and 72-96 h after dosing. For each collection interval, the urine volume was recorded and aliquots of the urine samples were collected and stored (frozen at $-20{ }^{\circ} \mathrm{C}$ ) until shipment to the analytical laboratory. Apixaban was quantitatively determined in plasma and urine samples by Intertek Pharmaceutical Services (El Dorado Hills, CA, USA), using validated high-performance liquid chromatographic-tandem mass spectrometric methods. The assay was performed on an AB Sciex $3000^{\mathrm{TM}}$ system using Analyst ${ }^{\circledR} 1.4$ software. Plasma and urine samples were extracted using protein precipitation and solid-phase extraction, respectively. The lower limit of quantification was $1.0 \mathrm{ng} / \mathrm{mL}$ for both apixaban plasma and urine assays. The standard curves were fitted by a $1 / \mathrm{x}^{2}$-weighted linear equation over the concentration range of $1.00-1,000 \mathrm{ng} / \mathrm{mL}$. The between-run and within-run variability, expressed as coefficients of variation, for apixaban in quality-control plasma samples were $\leq 9.49$ and $\leq 10.1 \%$, respectively, with deviations from the nominal concentration of no more than $\pm 16.2 \%$. The between-run and within-run variability for apixaban in quality-control urine samples were $\leq 3.65$ and $\leq 3.58 \%$, respectively, with deviations from the nominal concentration of no more than $\pm 12.4 \%$. All of the runs for the study met the acceptance criteria.

The international normalized ratio (INR), modified prothrombin time (mPT) and anti-Xa activity were measured to assess apixaban pharmacodynamic activity. Two $4.5 \mathrm{~mL}$ venous blood samples per time point were collected in $3.2 \%$ sodium citrate tubes and centrifuged for 10 min at $2,500 \times g$ at $4{ }^{\circ} \mathrm{C}$. The two plasma supernatants were pooled and then centrifuged again for $10 \mathrm{~min}$ at $2,500 \times g$ at $4{ }^{\circ} \mathrm{C}$ to minimize the platelet content in the plasma. Aliquots of the plasma sample were transferred into separate cryovials for INR, mPT and anti-Xa activity analysis and were stored at or below $-20{ }^{\circ} \mathrm{C}$ for shipment to the analytical laboratories.
The INR was measured by MDS (Toronto, ON, Canada), using an MLA 1600/1800 coagulation analyser and Hemoliance ${ }^{\circledR}$ RecombiPlasTin reagent (Beckman Coulter, Inc., Brea, CA, USA), having an international sensitivity index of $\sim 1.0$ (normal range $0.9-1.3$ ), with results reported to one decimal point. The $\mathrm{mPT}$ was measured by a validated method at Covance Laboratories, Inc. (Chantilly, VA, USA), using an MLA 1600c coagulation analyser (assay range 7-600 s). For the mPT assay, the thromboplastin reagent (Dade Thromboplastin C Plus; Siemens Healthcare Diagnostics, Deerfield, IL, USA) was diluted 1:2.25 with $100 \mathrm{nmol} / \mathrm{L}$ calcium chloride to decrease the rate of the clotting reaction [18]. Analyses of anti-Xa activity were performed by the Clinical Discovery Technologies group at Bristol-Myers Squibb (Hopewell, NJ, USA). Anti-Xa activity was measured using an $\mathrm{STA}^{\circledR}$ Rotachrom ${ }^{\circledR}$ heparin kit (Diagnostica Stago, Parsippany, $\mathrm{NJ}, \mathrm{USA}$ ) on an $\mathrm{STA}^{\circledR}-\mathrm{R}$ analyser [19]. Apixaban anti-Xa activity was calculated as the inverse of colour generation measured at $405 \mathrm{~nm}$ and interpolated against a 3-point linear kit LMWH calibration curve [range 0-2 IU/mL (LMWH units)] and against a 9-point log-linear apixaban calibration curve [range 7.8-2,000 ng/mL (apixaban units)].

\subsection{Pharmacokinetic and Pharmacodynamic Assessments}

Single-dose pharmacokinetic parameters were calculated from the individual plasma apixaban concentration-time data, using noncompartmental methods, by Kinetica ${ }^{\circledR}$ (version 4.2; InnaPhase Corp., Philadelphia, PA, USA) within the eToolbox Software package (EP version 2.4; Thermo Electron Corporation, Philadelphia, PA, USA). The parameters included $C_{\max }$, the time of $C_{\max }\left(t_{\max }\right)$, $\mathrm{AUC}_{\infty}$, AUC from time zero to the time of the last measurable concentration $\left(\mathrm{AUC}_{\text {last }}\right)$ and $t_{1 / 2}$. If the extrapolated AUC was greater than $20 \%$ of the subject's $\mathrm{AUC}_{\infty}$, the subject was excluded from summaries of $\mathrm{AUC}_{\infty}$ and $t_{1 / 2}$. $\mathrm{CL}_{\mathrm{R}}$ was calculated as the total cumulative amount of drug recovered in the urine, divided by $\mathrm{AUC}_{\text {last }}$ over the same time interval. The apparent total clearance after oral administration $(\mathrm{CL} / \mathrm{F})$ was calculated as dose divided by $\mathrm{AUC}_{\infty}$. The apparent volume of distribution at steady state $\left(V_{\mathrm{ss}} / \mathrm{F}\right)$ was calculated as dose $\times \mathrm{AUMC} / \mathrm{AUC}^{2}$, where AUMC is the area under the first-moment curve (the area under the first moment of the plasma concentration-time curve).

The mean pharmacodynamics-time profiles (INR, mPT and anti-Xa activity) for the age and sex groups were derived from individual subject data within each group, using the measurements collected from predose to $96 \mathrm{~h}$ postdose. Maximum values were determined for the INR, 
mPT and anti-Xa activity from the mean data. The AUC from time zero to $24 \mathrm{~h}$ postdose $\left(\mathrm{AUC}_{24}\right)$ was calculated for the INR and $\mathrm{mPT}$ from individual subject data. Given that an exploratory anti-Xa activity method was used in this study and not optimized for measurement of apixaban, AUC for anti-Xa activity was not calculated.

\subsection{Statistical Analysis}

\subsubsection{Pharmacokinetics}

Summary statistics were presented by group for all apixaban parameters $\left(C_{\max }, \mathrm{AUC}_{\infty}, \mathrm{AUC}_{\text {last }}, t_{\max }, t_{1 / 2}, \mathrm{CL} / \mathrm{F}\right.$, $V_{\mathrm{ss}} / \mathrm{F}$ and $\mathrm{CL}_{\mathrm{R}}$ ). Geometric means and coefficients of variation were provided for $C_{\max }, \mathrm{AUC}_{\infty}$ and $\mathrm{AUC}_{\text {last }}$. The median and range were presented for $t_{\max }$. Arithmetic means and standard deviations (SDs) were provided for $t_{1 / 2}$, $\mathrm{CL} / \mathrm{F}, V_{\mathrm{ss}} / \mathrm{F}$ and $\mathrm{CL}_{\mathrm{R}}$.

To assess the effects of age and sex on the pharmacokinetics of apixaban, analysis of variance was performed on $\ln \left(\mathrm{AUC}_{\infty}\right)$ and $\ln \left(C_{\max }\right)$. The factors in the analyses were age, sex and the interaction of age and sex. Point estimates and $90 \%$ CIs for means and for differences between means on a log scale were exponentiated to obtain estimates of geometric means and ratios of geometric means on the original scale. No adjustment was made for multiplicity. Absence of an age or sex effect on apixaban $\mathrm{AUC}_{\infty}$ or $C_{\max }$ was concluded if the corresponding $90 \%$ CI for the elderly to young ratio or for the female to male ratio of the population geometric mean was contained within an equivalence interval of 80-125\%. Regression analyses were conducted to assess potential relationships between apixaban $\mathrm{CL} / \mathrm{F}$ and $\mathrm{CL}_{\mathrm{R}}$ and creatinine clearance (CLcr), apixaban exposure ( $\mathrm{AUC}_{\infty}$ and $C_{\max }$ ) and CLcr, body weight and BMI.

\subsubsection{Pharmacodynamics}

Pharmacodynamic assessment-time profiles (means \pm SDs) were presented by age and sex groups for INR, $\mathrm{mPT}$ and anti-Xa activity values (expressed in both LMWH and apixaban units). Scatter plots of INR, mPT and anti-Xa activity values versus the plasma apixaban concentration were provided. The slope and intercept (90\% CI) were provided for INR, mPT and anti-Xa activity values (expressed in $\mathrm{ng} / \mathrm{mL}$ and LMWH units) based on the model-based regression analysis. SAS/ STAT $^{\circledR}$ version 8.2 software was used to perform the statistical analyses of the pharmacokinetic and pharmacodynamic data.

\subsection{Safety Assessments}

Subjects were closely monitored for adverse events throughout the study. The incidence of adverse events was tabulated, and all adverse events were reviewed for their relation to apixaban and clinical importance. Blood and urine samples for clinical laboratory testing were collected under fasting conditions at screening, at baseline, on day 1 , on day 2 and before study discharge. In addition, 12-lead electrocardiograms and physical examinations were performed at screening, at baseline and before study discharge.
Table 1 Demographic characteristics and physical measurements at baseline

$B M I$ body mass index, $C L c r$ creatinine clearance, $\mathrm{SCr}$ serum creatinine, $S D$ standard deviation

a $18-40$ years of age

b $\geq 65$ years of age

c CLcr is based on the Cockcroft-Gault calculation at screening: male CLcr $=[140-$ age $(\mathrm{y})] \times$ weight $(\mathrm{kg}) /$ $[72 \times \mathrm{SCr}(\mathrm{mg} / \mathrm{dL})]$; female $\mathrm{CLcr}=0.85 \times$ male CLcr

\begin{tabular}{|c|c|c|c|c|}
\hline \multirow[t]{2}{*}{ Characteristic } & \multicolumn{2}{|l|}{ Young $^{\mathrm{a}}$} & \multicolumn{2}{|l|}{ Elderly $^{\mathrm{b}}$} \\
\hline & Male $(n=20)$ & Female $(n=20)$ & Male $(n=20)$ & Female $(n=19)$ \\
\hline \multicolumn{5}{|l|}{ Age, years } \\
\hline Mean (SD) & $31(6)$ & $34(5)$ & $71(5)$ & $69(3)$ \\
\hline Range & $21-40$ & $21-40$ & $65-79$ & $65-76$ \\
\hline \multicolumn{5}{|l|}{ Race, $n(\%)$} \\
\hline White & $15(75)$ & $18(90)$ & $19(95)$ & $16(84)$ \\
\hline Black & $4(20)$ & $2(10)$ & 0 & $3(16)$ \\
\hline Hispanic & $1(5)$ & 0 & $1(5)$ & 0 \\
\hline \multicolumn{5}{|l|}{ Weight, kg } \\
\hline Mean (SD) & $76.8(12.4)$ & $65.3(8.9)$ & $78.9(12.0)$ & $70.2(10.8)$ \\
\hline Range & $60.0-97.0$ & $53.7-86.0$ & $55.0-114.0$ & $54.0-89.0$ \\
\hline \multicolumn{5}{|l|}{ BMI, $\mathrm{kg} / \mathrm{m}^{2}$} \\
\hline Mean (SD) & $26.1(3.5)$ & $25.4(3.1)$ & $28.3(2.8)$ & $28.4(2.9)$ \\
\hline Range & $20.2-31.9$ & $20.6-32.0$ & $21.0-31.9$ & $22.5-32.0$ \\
\hline \multicolumn{5}{|l|}{ CLcr, $\mathrm{mL} / \mathrm{min}^{\mathrm{c}}$} \\
\hline Mean (SD) & $123.3(23.0)$ & $115.7(15.0)$ & $82.8(20.8)$ & $75.1(12.4)$ \\
\hline Range & $87-179$ & $92-152$ & $62-144$ & $60-104$ \\
\hline
\end{tabular}




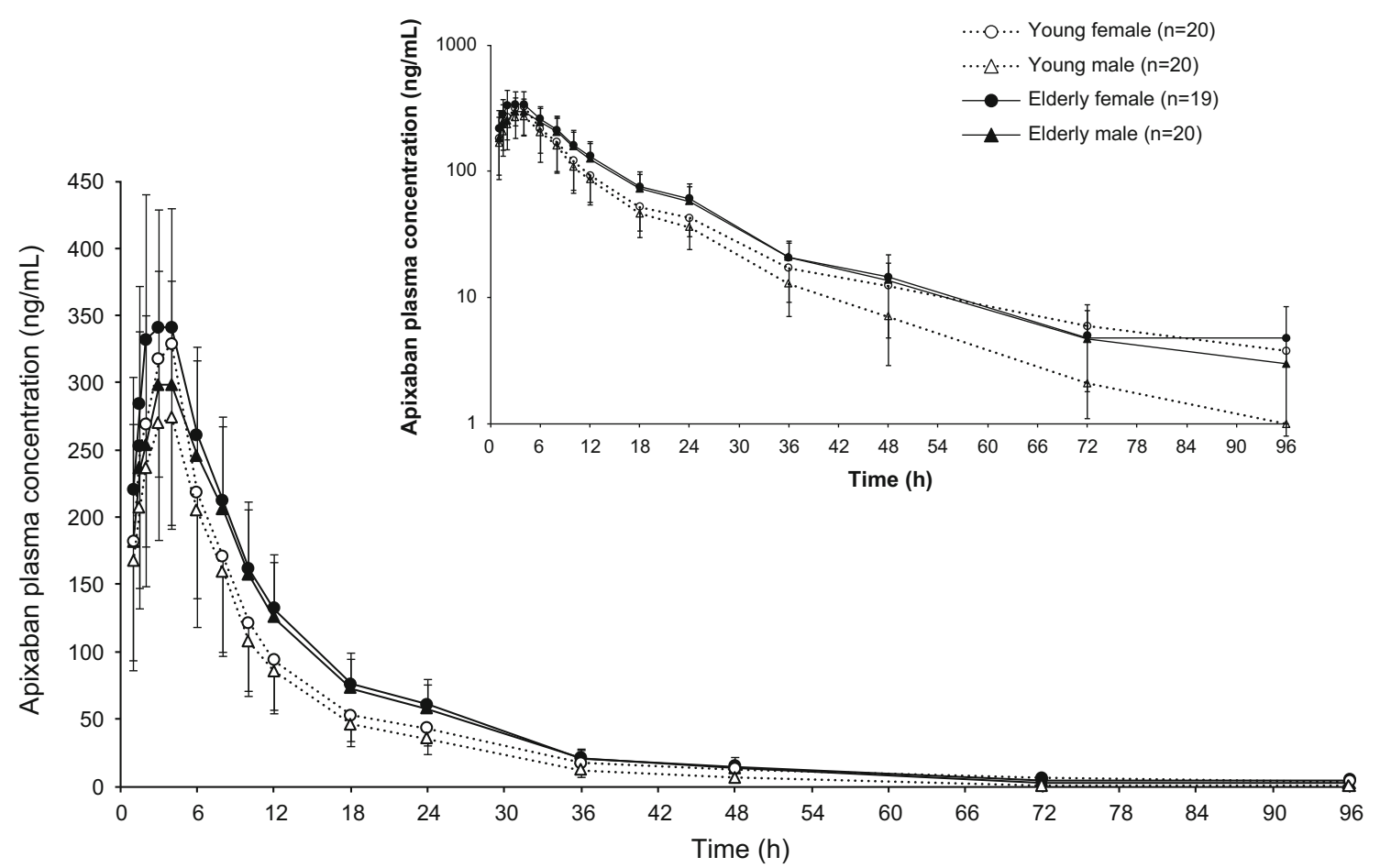

Fig. 1 Mean ( \pm standard deviation) plasma concentration-time profiles of apixaban after single-dose administration $(20 \mathrm{mg})$, by age and sex groups. The error bars represent \pm 1 standard deviation

Table 2 Apixaban pharmacokinetic parameters by age group

\begin{tabular}{|c|c|c|c|c|c|c|c|c|}
\hline & $\begin{array}{l}C_{\max }, \mathrm{ng} / \mathrm{mL}, \\
\mathrm{GM}(\mathrm{CV} \%)\end{array}$ & $\begin{array}{l}\mathrm{AUC}_{\infty}, \\
\mathrm{ng} \cdot \mathrm{h} / \mathrm{mL}, \\
\mathrm{GM}(\mathrm{CV} \%)\end{array}$ & $\begin{array}{l}\mathrm{AUC}_{\text {last }}, \\
\mathrm{ng} \cdot \mathrm{h} / \mathrm{mL}, \\
\mathrm{GM}(\mathrm{CV} \%)\end{array}$ & $\begin{array}{l}t_{\max }, \mathrm{h}, \\
\text { median } \\
(\min , \max )\end{array}$ & $\begin{array}{l}t_{1 / 2}, \mathrm{~h}, \\
\text { mean } \\
(\mathrm{SD})\end{array}$ & $\begin{array}{l}\mathrm{CL}_{\mathrm{R}}, \mathrm{L} / \mathrm{h}, \\
\text { mean }(\mathrm{SD})\end{array}$ & $\begin{array}{l}\mathrm{CL} / \mathrm{F}, \mathrm{L} / \mathrm{h}, \\
\text { mean }(\mathrm{SD})\end{array}$ & $\begin{array}{l}V_{\mathrm{ss}} / \mathrm{F}, \mathrm{L}, \\
\text { mean }(\mathrm{SD})\end{array}$ \\
\hline Young $(n=40)^{\mathrm{a}}$ & $315.4(34)$ & $3,424(32)$ & $3,360(32)$ & $3.0(1.0,4.0)$ & $11.98(5.15)^{\mathrm{b}}$ & $0.63(0.33)$ & $6.10(1.81)$ & $85.2(28.2)$ \\
\hline Elderly $(n=39)^{\mathrm{c}}$ & $336.9(27)$ & $4,536(23)$ & $4,451(23)$ & $3.0(1.0,4.0)$ & $15.45(7.39)$ & $0.45(0.13)$ & $4.52(0.98)$ & $77.5(27.0)$ \\
\hline $\begin{array}{l}\text { Ratio of adjusted } \\
\text { GM, elderly to } \\
\text { young }(90 \% \mathrm{CI})\end{array}$ & $1.07(0.96-1.19)$ & $1.32(1.20-1.46)$ & & & & & & \\
\hline
\end{tabular}

$A U C_{\infty}$ area under the plasma concentration-time curve from time zero extrapolated to infinite time, $A U C_{\text {last }}$ area under the plasma concentration-time curve from time zero to the time of the last measurable concentration, $C I$ confidence interval, $C L / F$ apparent total clearance after oral administration, $C L_{R}$ renal clearance, $C_{\max }$ maximum observed plasma concentration, $C V$ coefficient of variation, $G M$ geometric mean, $\max$ maximum, min minimum, $S D$ standard deviation, $t_{1 / 2}$ plasma terminal half-life, $t_{\max }$ time of $C_{\max }, V_{s s} / F$ apparent volume of distribution at steady state

a $18-40$ years of age

${ }^{\mathrm{b}}$ One young female subject was excluded from the summary statistics (and related analyses) for AUC $\mathrm{\infty}_{\infty}$ and $t_{1 / 2}$ on the basis of predefined criteria c $\geq 65$ years of age

\section{Results}

\subsection{Study Population}

A total of 79 subjects met the study criteria, were treated and completed the study. The clinical phase of the study was conducted from May to November 2005. The baseline characteristics of all subjects are summarized in Table 1 . The mean age was 71 years for elderly males and 69 years for elderly females, versus 31 and 34 years for young males and young females, respectively.

\subsection{Pharmacokinetics}

The mean plasma concentration-time profiles of apixaban for the four age and sex groups are shown in Fig. 1. Pharmacokinetic parameters and the results of statistical analyses of $C_{\max }$ and $\mathrm{AUC}_{\infty}$ by age and sex are shown in 
Table 3 Apixaban pharmacokinetic parameters by sex group

\begin{tabular}{|c|c|c|c|c|c|c|c|c|}
\hline & $\begin{array}{l}C_{\max }, \\
\text { ng/mL, } \\
\text { GM }(\mathrm{CV} \%)\end{array}$ & $\begin{array}{l}\mathrm{AUC}{ }_{\infty}, \\
\mathrm{ng} \cdot \mathrm{h} / \mathrm{mL}, \\
\mathrm{GM}(\mathrm{CV} \%)\end{array}$ & $\begin{array}{l}\mathrm{AUC}_{\text {last }}, \\
\mathrm{ng} \cdot \mathrm{h} / \mathrm{mL}, \\
\mathrm{GM}(\mathrm{CV} \%)\end{array}$ & $\begin{array}{l}t_{\max }, \mathrm{h}, \\
\text { median } \\
(\min , \max )\end{array}$ & $\begin{array}{l}t_{1 / 2}, \mathrm{~h}, \\
\text { mean }(\mathrm{SD})\end{array}$ & $\begin{array}{l}\mathrm{CL}_{\mathrm{R}}, \mathrm{L} / \mathrm{h}, \\
\text { mean }(\mathrm{SD})\end{array}$ & $\begin{array}{l}\mathrm{CL} / \mathrm{F}, \mathrm{L} / \mathrm{h}, \\
\text { mean }(\mathrm{SD})\end{array}$ & $\begin{array}{l}V_{\mathrm{ss}} / \mathrm{F}, \mathrm{L}, \\
\text { mean }(\mathrm{SD})\end{array}$ \\
\hline Female $(n=39)$ & $353.4(31)$ & $4,235(28)^{\mathrm{a}}$ & $4,116(29)$ & $3.0(1.0,4.0)$ & $14.89(7.06)^{\mathrm{a}}$ & $0.46(0.18)$ & $4.91(1.41)$ & 79.7 (28.8) \\
\hline Male $(n=40)$ & $301.0(26)$ & $3,680(30)$ & $3,626(30)$ & $3.0(1.0,4.0)$ & $12.60(5.92)$ & $0.62(0.31)$ & $5.69(1.79)$ & $82.8(26.9)$ \\
\hline $\begin{array}{l}\text { Ratio of adjusted } \\
\text { GM, female to } \\
\text { male }(90 \% \text { CI) }\end{array}$ & $1.18(1.06-1.31)$ & $1.15(1.04-1.27)$ & & & & & & \\
\hline
\end{tabular}

$A U C_{\infty}$ area under the plasma concentration-time curve from time zero extrapolated to infinite time, $A U C_{\text {last }}$ area under the plasma concentration-time curve from time zero to the time of the last measurable concentration, $C I$ confidence interval, $C L / F$ apparent total clearance after oral administration, $C L_{R}$ renal clearance, $C_{\max }$ maximum observed plasma concentration, $C V$ coefficient of variation, $G M$ geometric mean, $\max$ maximum, min minimum, $S D$ standard deviation, $t_{1 / 2}$ plasma terminal half-life, $t_{\max }$ time of $C_{\max }, V_{s s} / F$ apparent volume of distribution at steady state

${ }^{a}$ One young female subject was excluded from the summary statistics (and related analyses) for $\mathrm{AUC}_{\infty}$ and $t_{1 / 2}$ on the basis of predefined criteria

Tables 2 and 3 , respectively. Apixaban reached $C_{\max }$ within $3 \mathrm{~h}$ of administration, on average, regardless of age or sex. Age had no effect on apixaban $C_{\max }$, whereas older age was associated with a $32 \%$ greater apixaban $\mathrm{AUC}_{\infty}$, compared with younger age. In female subjects, the apixaban geometric mean $C_{\max }$ and $\mathrm{AUC}_{\infty}$ values were 18 and $15 \%$ higher, respectively, than the values in male subjects. The age by sex interaction was nonsignificant for $C_{\max }$ and $\operatorname{AUC}_{\infty}(p=0.94$ and $p=0.40$, respectively).

The apixaban $\mathrm{CL}_{\mathrm{R}}$ tended to be lower in elderly subjects $(0.45 \mathrm{~L} / \mathrm{h})$ than in young subjects $(0.63 \mathrm{~L} / \mathrm{h})$ and lower in female subjects $(0.46 \mathrm{~L} / \mathrm{h})$ than in male subjects $(0.62 \mathrm{~L} /$ h); these differences in $C_{R}$ also appeared to be reflected in $\mathrm{CL} / \mathrm{F}$ (young versus elderly 6.1 versus $4.5 \mathrm{~L} / \mathrm{h}$; male versus female 5.7 versus $4.9 \mathrm{~L} / \mathrm{h}$ ). The mean apixaban $t_{1 / 2}$ in young male subjects was approximately $10 \mathrm{~h}$, whereas in young female and elderly subjects it ranged from 14 to $16 \mathrm{~h}$. A weak increasing linear correlation was observed between apixaban CL/F and CLcr $\left(R^{2}=0.2674, \quad p<0.0001\right.$; Fig. 2a) and between $\mathrm{CL}_{\mathrm{R}}$ and CLcr $\left(R^{2}=0.1773\right.$, $p<0.0001$; Fig. 2b). Among the relationships between apixaban exposure $\left(\mathrm{AUC}_{\infty}\right.$ and $\left.C_{\max }\right)$ and other factors (body weight and BMI) that were also explored, only apixaban $C_{\max }$ versus body weight showed a statistically significant $(p=0.009)$ but weak $\left(R^{2}=0.09\right)$ negative linear correlation.

\subsection{Pharmacodynamics}

Data from 39 subjects (6 young males, 12 young females, 11 elderly males and 10 elderly females) were included in the summary and comparisons of apixaban pharmacodynamics. Pharmacodynamic data from the first 40 subjects enrolled in the study were excluded because of errors with sample collection and processing techniques (such as inadequate mixing of the sample, sustained application of the tourniquet and repeated venepuncture in the same location), which led to spuriously elevated clotting time assessments regardless of age, sex or the plasma apixaban concentration. Sample collection and processing techniques have been shown to influence clotting time measurements $[20,21]$. Following retraining of the clinical site on adequate mixing of blood with anticoagulant in the sample tube and timely sample transfer, etc., clotting time measurements showed greater consistency with prior experience $[7,9]$.

The time course of the mean INR, mPT and anti-Xa activity tracked the plasma concentration-time curve of apixaban (Fig. 3), with maximum values for all pharmacodynamic endpoints observed around the apixaban $t_{\max }$ $(3 \mathrm{~h})$. Consistent with the pharmacokinetic profile in elderly and young subjects, elderly subjects generally had higher mean INR, mPT and anti-Xa values than young subjects over the observed time course, but the differences in the maximum mean (SD) INR, anti-Xa activity and $\mathrm{mPT}$ between age groups were less than $15 \%$ : INR, $1.55(0.18)$ and 1.46 (0.19); anti-Xa activity, 275 (75) and 295 (91) ng/ $\mathrm{mL} ; \mathrm{mPT}, 149$ (25) and 130 (26) s in the elderly and young groups, respectively. Similarly, the differences in the mean (SD) $\mathrm{AUC}_{24}$ between age groups for these INR and $\mathrm{mPT}$ assessments were less than $20 \%$ : INR, 31.5 (2.23) and 29.3 (2.09); mPT, 2,604 (378) and 2,137 (295) s.h in the elderly and young groups, respectively.

The mean INR, mPT and anti-Xa activity profiles in female subjects did not reflect the slightly higher apixaban exposure observed in that group relative to male subjects, with mean INR and $\mathrm{mPT}$ values being slightly lower in females than in males, and no discernible difference in anti-Xa. There were no substantial differences at maximal values; the differences between sex groups were less than $15 \%$ at maximum mean (SD) values: INR, $1.47(0.12)$ and 1.56 (0.16); mPT, 132 (23) and 150 (33) s; anti-Xa activity, 292 (96) and $274(60) \mathrm{ng} / \mathrm{mL}$ in female and male subjects, respectively (Fig. 3). Furthermore, the differences in the mean (SD) $\mathrm{AUC}_{24}$ between the sexes for these INR and mPT assessments were less than $15 \%$ : INR, 29.7 (1.58) 


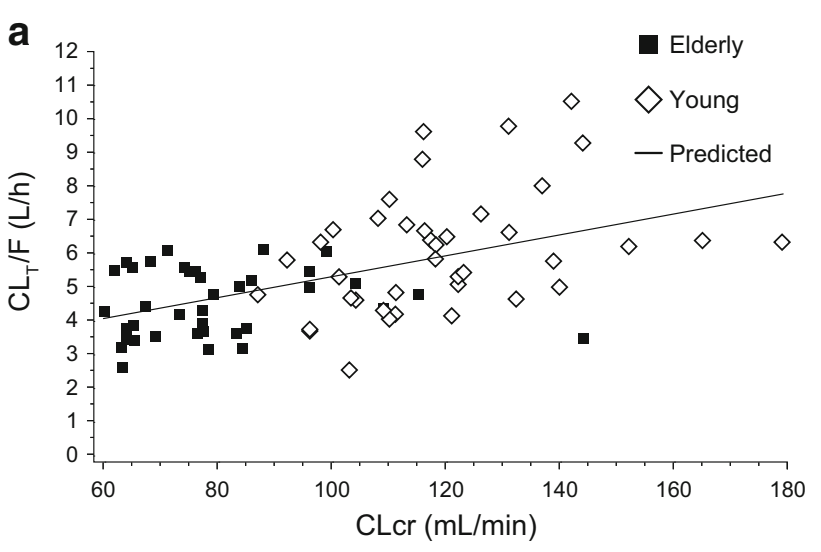

Regression equation: $\mathrm{CL}_{T} / \mathrm{F}=2.231+0.031 \times \mathrm{CLcr}$ $\mathrm{R}^{2}=0.2674, \mathrm{p}$-value $<0.0001$

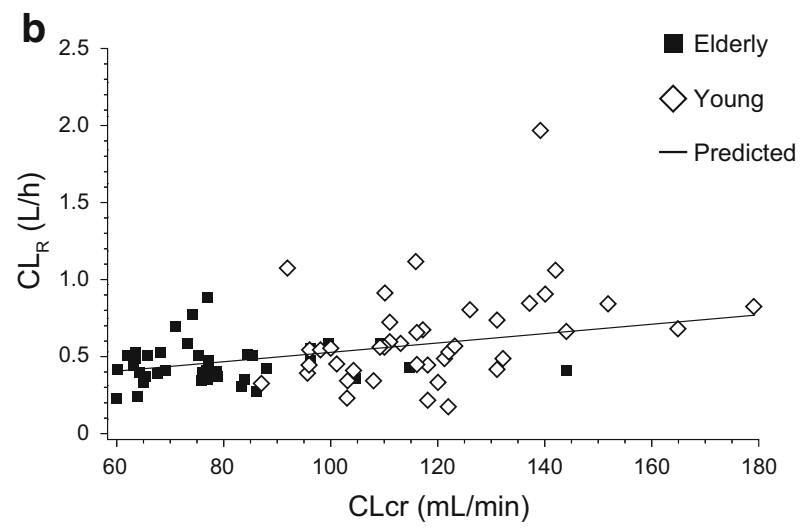

Regression equation: $\mathrm{CL}_{\mathrm{R}}=0.21075+0.00313 \times \mathrm{CLCr}$ $\mathrm{R}^{2}=0.1773, \mathrm{p}$-value $<0.0001$

Fig. 2 Linear regression of a apparent total clearance after oral administration $(C L / F)$ and $\mathbf{b}$ renal clearance $\left(C_{R}\right)$ of apixaban versus creatinine clearance (CLcr), by age groups. All subjects received apixaban $20 \mathrm{mg}$. For $\mathrm{CL}_{\mathrm{R}}$, one patient with a $\mathrm{CL}_{\mathrm{R}}$ of $2.08 \mathrm{~L} / \mathrm{h}$ was excluded from the statistical analysis

and 31.5 (2.91); mPT, 2,248 (296) and 2,570 (476) s.h in female and male subjects, respectively. These findings likely reflect limitations in the sensitivity of the respective pharmacodynamic assays and their ability to accurately detect small differences in apixaban exposure; however, the anti-Xa activity appeared to better reflect the observed apixaban concentration-time profiles of females and males.

There were no obvious differences in the plasma concentration-pharmacodynamic response relationships of apixaban (Fig. 4a, b) across age or sex. The slope and intercept $(90 \% \mathrm{CI})$ for the INR versus the apixaban concentration were $0.0021(0.0017-0.0026) \mathrm{mL} / \mathrm{ng}$ and 1.09 (1.07-1.12), respectively; the slope and intercept (90\% CI) for the mPT versus the apixaban concentration were 0.31 $(0.28-0.33) \mathrm{mL} \cdot \mathrm{s} / \mathrm{ng}$ and $61.7(58.9-64.5) \mathrm{s}$, respectively. A positive linear relationship was observed between individual anti-Xa activity [expressed in apixaban units (ng/ $\mathrm{mL})$ ] and the corresponding plasma apixaban concentrations (Fig. 4c). The slope and intercept (90\% CI) for anti-Xa activity (expressed in $\mathrm{ng} / \mathrm{mL}$ ) versus the apixaban concentration were $0.85(0.83-0.86)$ and 13.0 (8.7-17.2) $\mathrm{ng} / \mathrm{mL}$, respectively. When anti-Xa activity was expressed in LMWH units of $\mathrm{IU} / \mathrm{mL}$, based on the kit 3-point standard curve $(0-2 \mathrm{IU} / \mathrm{mL})$, a similar linear relationship was observed over a narrower plasma apixaban concentration range (approximately $0-200 \mathrm{ng} / \mathrm{mL}$, as shown in Fig. 4d). The slope and intercept (90\% CI) for anti-Xa activity (expressed in IU/mL) versus the apixaban concentration were $0.0134(0.0130-0.0138) \mathrm{IU} / \mathrm{ng}$ and $0.048(0.023-0.072) \mathrm{IU} / \mathrm{mL}$, respectively. The relationship between anti-Xa activity and the plasma apixaban concentration was less variable than that observed for the INR and mPT (Fig. 4a-d).

\subsection{Safety and Tolerability}

A single $20 \mathrm{mg}$ dose of apixaban was well tolerated by all subjects in this study; there were no serious adverse events, significant bleeding-related adverse events or discontinuations due to adverse events. A total of 21 adverse events were reported by 16 subjects; all were considered to be of mild or moderate intensity. Seven subjects (including six females) reported minor bleeding-related adverse events, including three subjects with positive faecal occult blood (one young female, one elderly female and one elderly male), three subjects with ecchymosis (two young females and one elderly female) and one subject (an elderly female) with a haematoma secondary to venepuncture. All bleeding events were mild in intensity and generally resolved within 1-7 days. The remaining adverse events consisted of eczema, pruritus, headache, dizziness, iron deficiency anaemia, blood draw site pain, cholelithiasis with elevated transaminases, subcutaneous abscess, pharyngitis, cheilitis and constipation, each occurring in one subject except for headache (two subjects).

\section{Discussion}

Apixaban is approved for prevention of VTE following elective knee or hip replacement surgery and for stroke prevention in patients with NVAF; these patient populations tend to comprise elderly men and women [22-25]. Therefore, it is important to understand if age- or sexrelated differences influence the pharmacokinetics and pharmacodynamics of agents used in the management of these patients.

In this study, a single oral $20 \mathrm{mg}$ dose of apixaban was safe and well tolerated in all subjects, regardless of age or sex. A $20 \mathrm{mg}$ dose was chosen for this study because the systemic exposure from a single $20 \mathrm{mg}$ dose is 

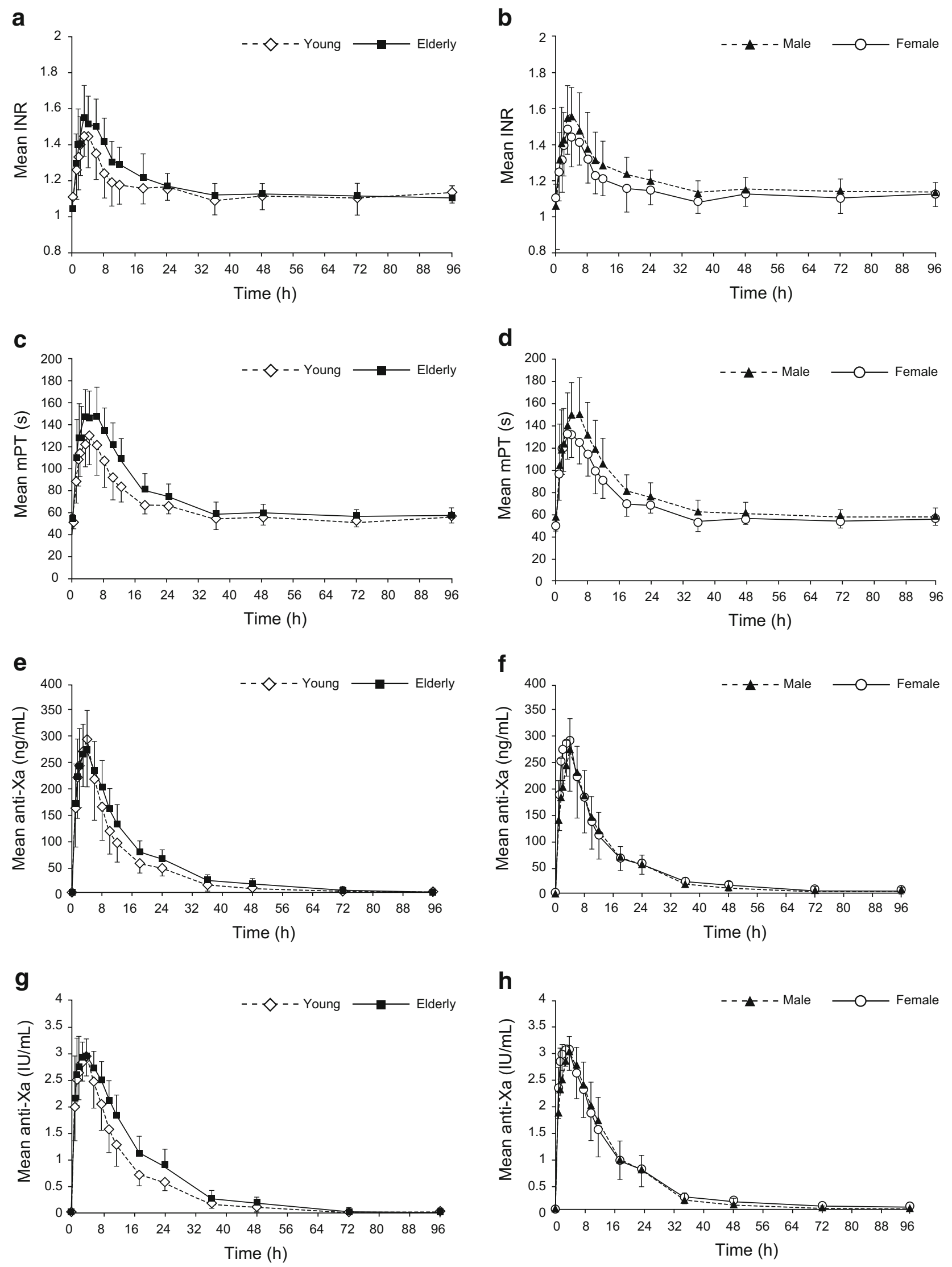
Fig. 3 a, b International normalized ratio (INR), c, d modified prothrombin time (mPT), e, f anti-Xa activity (expressed in $\mathrm{ng} / \mathrm{mL}$ ), and $\mathbf{g}, \mathbf{h}$ anti-Xa activity (expressed in $\mathrm{IU} / \mathrm{mL}$ ) versus time, by age (young 18-40 years of age; elderly $\geq 65$ years of age) and sex groups. The error bars represent \pm 1 standard deviation

representative of the steady-state exposure achieved with the highest dose (10 mg twice daily) used in the apixaban phase 3 clinical trials. Apixaban exposure was approximately $32 \%$ higher in elderly subjects than in young subjects and $15 \%$ greater in female subjects than in male subjects, indicating that age- and sex-related influences have a small to modest impact on apixaban exposure. Ageand sex-related influences on pharmacokinetics have been attributed to multiple factors [10], several of which were

a
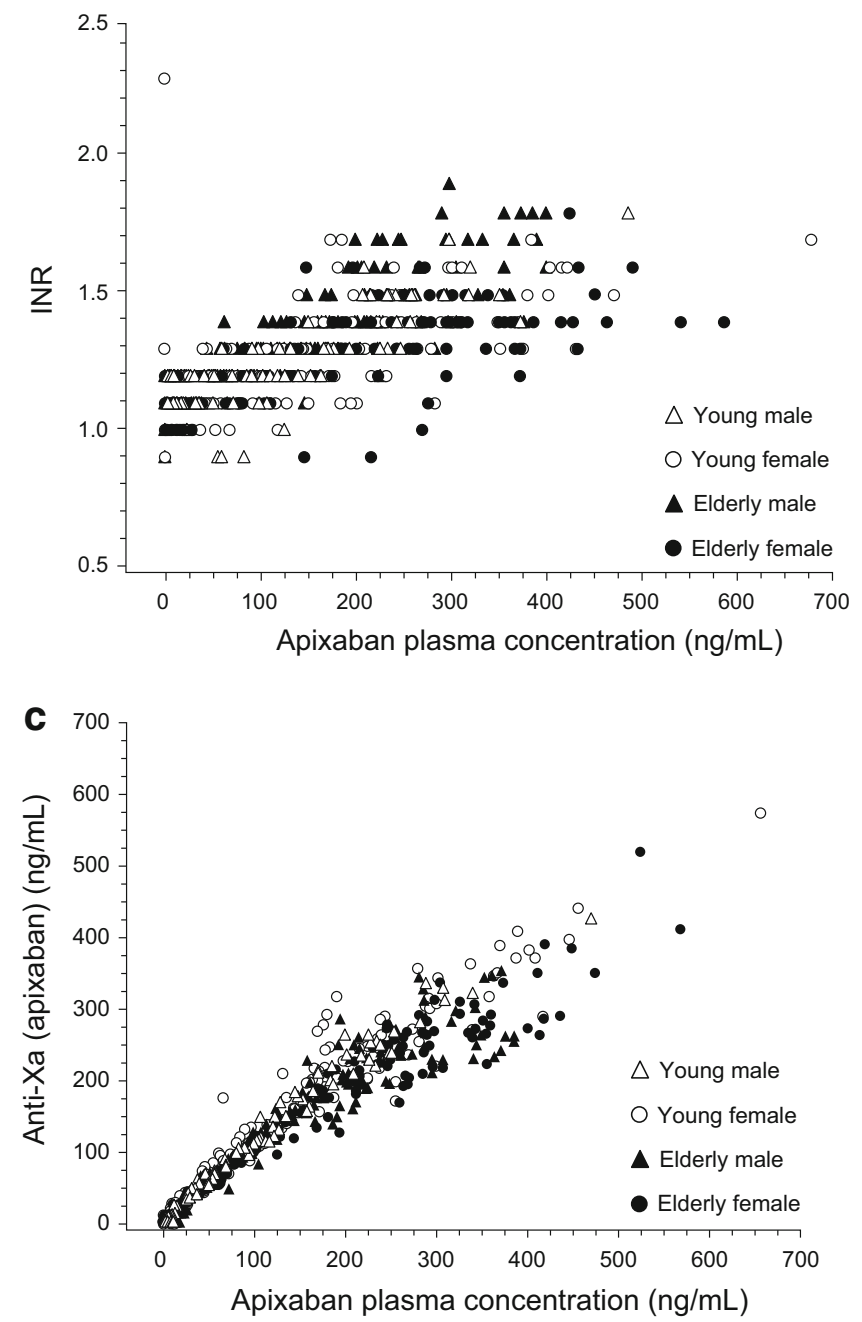

Fig. 4 Scatter plots of a the individual international normalized ratio (INR), $\mathbf{b}$ the individual modified prothrombin time (mPT), $\mathbf{c}$ individual anti-Xa activity levels (expressed in $\mathrm{ng} / \mathrm{mL}$ of apixaban) and d individual anti-Xa activity levels [expressed in $\mathrm{IU} / \mathrm{mL}$ of low molecular weight heparin (LMWH)] versus plasma apixaban explored in this study, including associations between apixaban exposure and renal function, body weight and BMI. Among these factors, the strongest trend was observed between apixaban exposure and renal function; therefore, the relationship between apixaban CL/F and renal function was explored further.

$\mathrm{CL}_{\mathrm{R}}$ accounts for approximately $27 \%$ of total clearance; therefore, a relationship between apixaban exposure and renal function would be expected. In this study, $\mathrm{CL}_{\mathrm{R}}$ and CL/F tended to be lower in elderly subjects than in young subjects and lower in female subjects than in male subjects. There was a direct relationship between apixaban clearance and CLcr, which was consistent with the observed inverse relationship between apixaban exposure

b

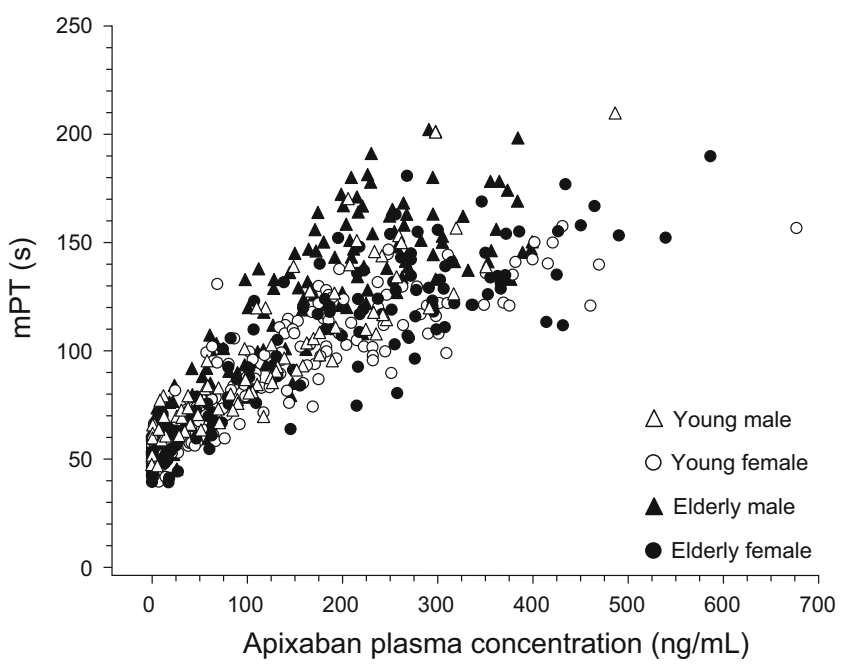

d

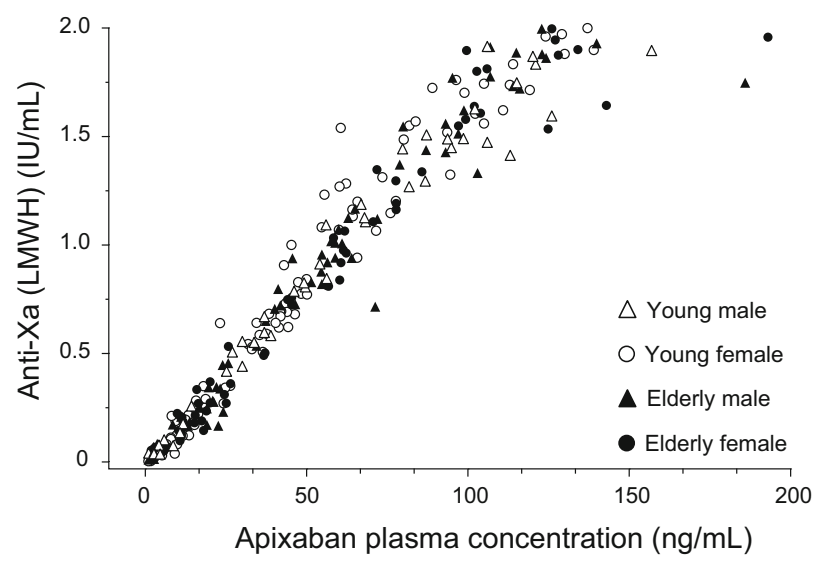

concentrations, by age (young: 18-40 years of age; elderly: $\geq 65$ years of age) and sex groups. Data points that are above the upper limit of the anti-Xa activity assay range ( $2 \mathrm{IU} / \mathrm{mL}$, corresponding to an apixaban concentration of $\sim 200 \mathrm{ng} / \mathrm{mL}$ ) are not included in the figure 
and CLcr. These trends suggest that differences in renal function may have contributed to the differences in apixaban $C_{R}$ therefore reflected in the $C L / F$ and exposure observed between groups. While determining the effect of renal function on apixaban pharmacokinetics was not an aim of this study, the effect of renal impairment on apixaban pharmacokinetics has been evaluated in two other studies - one in subjects with mild, moderate and severe renal impairment, and another in subjects with end-stage renal disease on haemodialysis $[26,27]$. The results from those two studies indicated that mild and moderate renal impairment had a modest effect on the apixaban AUC; the apixaban AUC was approximately $40 \%$ greater in subjects with severe renal impairment or end-stage renal disease $[26,27]$. Given that the range of CLcr in the current study was $60-179 \mathrm{~mL} / \mathrm{min}$, consistent with the age range of the subjects, the difference in $\mathrm{CL}_{\mathrm{R}}$ would partly contribute to the observed differences in exposure between groups. The apixaban $t_{1 / 2}$ also appeared to be longer in elderly and female subjects (elderly versus young 15.45 versus $11.98 \mathrm{~h}$; female versus male 14.89 versus $12.60 \mathrm{~h}$ ).

As noted, since only weak relationships were observed between apixaban exposure and body weight and BMI, the relationship between apixaban clearance and these two demographic factors were not explored further in this study. A study evaluating the impact of body weight on apixaban pharmacokinetics has shown that apixaban exposure is approximately 30 higher and $22 \%$ lower in individuals weighing $<50 \mathrm{~kg}$ or $>120 \mathrm{~kg}$, respectively, compared with subjects weighing $65-85 \mathrm{~kg}$ [27]. Therefore, given the limited range of body weight in this study $(54-114 \mathrm{~kg})$, it is not surprising that a weak association between apixaban exposure and body weight and BMI was observed.

As expected, changes in the pharmacodynamic measures of INR, mPT and anti-Xa activity over time closely followed the apixaban concentration-time profile. All three measures exhibited a positive linear relationship with the plasma apixaban concentration. Similar to what has been previously reported, the relationship between the plasma apixaban concentration and the INR and $\mathrm{mPT}$ is weaker and more variable than the relationship between the plasma apixaban concentration and anti-Xa activity [18]. This is expected, since endogenous factors contributing to clotting play roles in INR and mPT measurement, while the anti-Xa activity assay used in this study relies on the addition of exogenous bovine FXa, thus making the anti-Xa test more dependent on an FXa inhibitor (apixaban) concentration and less on other endogenous factors.

Measurement of anti-Xa activity using an apixaban standard curve showed a positive linear correlation over the full range of plasma apixaban concentrations (from 0 to $\sim 700 \mathrm{ng} / \mathrm{mL}$ ) observed in this study. Anti-Xa activity determined using the kit LMWH standards also showed a linear relationship, although over a narrower range of apixaban concentrations ( $\sim 0$ to $200 \mathrm{ng} / \mathrm{mL})$. It should be noted that this was the first clinical study in which apixaban anti-Xa activity was measured using the STA ${ }^{\circledR}$-Rotachrom ${ }^{\circledR}$ heparin kit. Given the use of exogenous bovine FXa in the Rotachrom ${ }^{\circledR}$ assay and the pharmacological characteristics of apixaban, the anti-Xa activity determined with this assay is expected to correlate well with apixaban concentrations. Therefore, any apparent discordance between anti-Xa activity and apixaban concentrations likely reflects assay limitations rather than a difference in the pharmacological effect of apixaban. On the basis of the results of this study, the STA ${ }^{\circledR}$-Rotachrom ${ }^{\circledR}$ method was further optimized for use in clinical trials and was used to establish a direct linear correlation over a wider concentration range between apixaban anti-Xa activity (expressed in $\mathrm{IU} / \mathrm{mL}$ ) and the plasma apixaban concentration (expressed in $\mathrm{ng} / \mathrm{mL}$ ) $[18,19]$. Therefore, anti-Xa activity may have utility as a bioassay for apixaban.

The small to modest effects of age and sex on apixaban pharmacokinetics and pharmacodynamics observed in this study are not likely to be clinically relevant, given the favourable benefit-risk profile of apixaban observed in phase 3 clinical trials. Elderly male and female patients were included in the apixaban phase 3 clinical trials for prevention of thromboembolism in knee and hip replacement surgery (mean age $\sim 63$ years; $\sim 40 \%$ males) and for stroke prevention in patients with AF (mean age $\sim 69$ years; 59-65\% males) [22-25, 29]. The $2.5 \mathrm{mg}$ twice-daily regimen was used in all phase 3 studies of apixaban in VTE prevention, irrespective of patient age or sex, and the efficacy and safety outcomes from those trials confirmed that no dose adjustment is needed for age or sex in that population [30]. The safety and efficacy profiles observed for apixaban in the subpopulation analyses of the phase 3 ARISTOTLE and AVERROES studies in patients with NVAF also indicated that dose adjustment for age or sex alone is not necessary [24, 25]. The ARISTOTLE and AVERROES trials did have prespecified dose modification criteria that included age as a component. Subjects with at least two out of three risk factors for bleeding (age $\geq 80$ years, body weight $\leq 60 \mathrm{~kg}$ and/or serum creatinine $\geq 1.5 \mathrm{mg} / \mathrm{dL}$ ) received apixaban $2.5 \mathrm{mg}$ twice daily rather than $5 \mathrm{mg}$ twice daily, which maintained the desired safety and efficacy profiles in patients who met these criteria $[24,25]$. The individual impact of these factors on apixaban exposure is modest and does not warrant dose modification. Nevertheless, when they exist in combination, the resulting increase in exposure may warrant a reduction in dose to mitigate the inherently higher risk of bleeding that may be present in some patient populations $[26-29,31]$. 


\section{Conclusion}

This study demonstrated that age- and sex-related influences on apixaban pharmacokinetics were small to modest and do not appear to be clinically meaningful on the basis of age and sex alone. Corresponding minor differences were also present in pharmacodynamic markers of anticoagulation activity. Anti-Xa activity showed a direct linear relationship with plasma apixaban concentrations, regardless of age or sex groups, and appeared to be less variable than other pharmacodynamic assessments, such as PT/INR. Apixaban was well tolerated by all subjects in this study. No dose adjustment is recommended for apixaban on the basis of age or sex alone. However, in the presence of multiple factors that increase apixaban exposure, dose adjustment may be warranted, such as in AF patients with at least two of these three risk factors for bleeding: age $\geq 80$ years, body weight $\leq 60 \mathrm{~kg}$ and/or serum creatinine $\geq 1.5 \mathrm{mg} / \mathrm{dL}$.

Acknowledgments This study was sponsored by Bristol-Myers Squibb Company and Pfizer Inc. The authors would like to thank Kenneth Moore and Alexander Bragat for their contributions to the conduct of the study, and Zhaoqing Wang for performing the anti-Xa activity test. Professional editorial support was provided by Dana Fox, $\mathrm{PhD}$, CMPP, and Andy Shepherd, PhD, of Caudex Medical, and was funded by Bristol-Myers Squibb and Pfizer.

Conflict of interest Drs. Frost, Song, Wang, Barrett, Schuster and LaCreta are employees of Bristol-Myers Squibb. Dr. Shenker was an employee of Bristol-Myers Squibb at the time when this research was conducted, and he is currently an employee of GlaxoSmithKline. Dr. Harris is an employee of Seaview Research, Miami, FL, USA.

Open Access This article is distributed under the terms of the Creative Commons Attribution Noncommercial License which permits any noncommercial use, distribution, and reproduction in any medium, provided the original author(s) and the source are credited.

\section{References}

1. Geerts WH, Bergqvist D, Pineo GF, et al. Prevention of venous thromboembolism: American College of Chest Physicians evidence-based clinical practice guidelines (8th edition). Chest. 2008;133(6 Suppl):381S-453S.

2. Hirsh J, Guyatt G, Albers GW, et al. Antithrombotic and thrombolytic therapy: American College of Chest Physicians evidence-based clinical practice guidelines (8th edition). Chest. 2008;133(6 Suppl):110S-2S.

3. Weitz JI, Hirsh J, Samama MM. New antithrombotic drugs: American College of Chest Physicians evidence-based clinical practice guidelines (8th edition). Chest. 2008;133(6 Suppl):234S$56 \mathrm{~S}$.

4. Ansell J, Hirsh J, Hylek E, et al. Pharmacology and management of the vitamin K antagonists: American College of Chest Physicians evidence-based clinical practice guidelines (8th edition). Chest. 2008;133(6 Suppl):160S-98S.
5. Palareti G, Manotti C, DAngelo A, et al. Thrombotic events during oral anticoagulant treatment: results of the inceptioncohort, prospective, collaborative ISCOAT study: ISCOAT Study Group (Italian Study on Complications of Oral Anticoagulant Therapy). Thromb Haemost. 1997;78(6):1438-43.

6. Raghavan N, Frost CE, Yu Z, et al. Apixaban metabolism and pharmacokinetics after oral administration to humans. Drug Metab Dispos. 2009;37(1):74-81.

7. Frost C, Wang J, Nepal S, et al. Apixaban, an oral, direct factor Xa inhibitor: single dose safety, pharmacokinetics, pharmacodynamics and food effect in healthy subjects. Br J Clin Pharmacol. 2013;75(2):476-87.

8. Wang L, Zhang D, Raghavan N, et al. In vitro assessment of metabolic drug-drug interaction potential of apixaban through cytochrome $\mathrm{P} 450$ phenotyping, inhibition, and induction studies. Drug Metab Dispos. 2010;38(3):448-58.

9. Frost C, Nepal S, Schuster A, et al. Safety, pharmacokinetics and pharmacodynamics of multiple oral doses of apixaban, a factor Xa inhibitor, in healthy subjects. Br $\mathrm{J}$ Clin Pharmacol. 2013;76(5):776-86.

10. Burton DG, Allen MC, Bird JL, et al. Bridging the gap: ageing, pharmacokinetics and pharmacodynamics. J Pharm Pharmacol. 2005;57(6):671-9.

11. Meibohm B, Beierle I, Derendorf H. How important are gender differences in pharmacokinetics? Clin Pharmacokinet. 2002;41(5):329-42.

12. Roller RE, Iglseder B, Dovjak P, et al. Oral anticoagulation and risk of bleeding in elderly patients - the aspect of polypharmacy. Wien Med Wochenschr. 2010;160(11-12):270-5.

13. Verma S, Kato S, Blum R, et al. Association of age, gender, and weight on maintenance dose of intravenous unfractionated heparin. Am J Hematol. 2010;85(8):624-6.

14. Volgman AS, Manankil MF, Mookherjee D, et al. Women with atrial fibrillation: greater risk, less attention. Gend Med. 2009;6(3):419-32.

15. Feinberg WM, Blackshear JL, Laupacis A, Kronmal R, Hart RG. Prevalence, age distribution, and gender of patients with atrial fibrillation: analysis and implications. Arch Intern Med. 1995;155(5):469-73.

16. Buller H, Deitchman D, Prins M, Segers A, Botticelli Investigators, Writing Committee. Efficacy and safety of the oral direct factor Xa inhibitor apixaban for symptomatic deep vein thrombosis. The Botticelli DVT dose-ranging study. J Thromb Haemost. 2008;6(8):1313-8.

17. Lassen MR, Davidson BL, Gallus A, et al. The efficacy and safety of apixaban, an oral, direct factor Xa inhibitor, as thromboprophylaxis in patients following total knee replacement. J Thromb Haemost. 2007;5(12):2368-75.

18. Barrett YC, Wang Z, Frost C, et al. Clinical laboratory measurement of direct factor Xa inhibitors: anti-Xa assay is preferable to prothrombin time assay. Thromb Haemost. 2010;104(6):1263-71.

19. Barrett YC, Wang J, Song Y, et al. A randomised assessment of the pharmacokinetic, pharmacodynamic and safety interaction between apixaban and enoxaparin in healthy subjects. Thromb Haemost. 2012;107(5):916-24.

20. Bates SM, Weitz JI. Coagulation assays. Circulation. 2005;112:e53-60.

21. Chitlur M. Challenges in the laboratory analyses of bleeding disorders. Thromb Res. 2012;130(1):1-6.

22. Lassen MR, Gallus A, Raskob GE, et al. Apixaban versus enoxaparin for thromboprophylaxis after hip replacement. N Engl J Med. 2010;363(26):2487-98.

23. Lassen MR, Raskob GE, Gallus A, et al. Apixaban versus enoxaparin for thromboprophylaxis after knee replacement 
(ADVANCE-2): a randomised double-blind trial. Lancet. 2010;375(9717):807-15.

24. Connolly SJ, Eikelboom J, Joyner C, et al. Apixaban in patients with atrial fibrillation. N Engl J Med. 2011;364(9):806-17.

25. Granger CB, Alexander JH, McMurray JJ, et al. Apixaban versus warfarin in patients with atrial fibrillation. $\mathrm{N}$ Engl $\mathrm{J}$ Med. 2011;365(11):981-92.

26. Shenker A, Wang J, Pursley J, Boyd RA, LaCreta F, Frost C. Apixaban pharmacokinetics and pharmacodynamics in subjects with renal impairment. In: Presented at: Annual Meeting of the American College of Clinical Pharmacology; September 23-25, 2012; San Diego. Clin Pharmacol Drug Dev. 2012;1(4):175-229.

27. Wang X, Song Y, Tirucherai G, et al. Apixaban pharmacokinetics in subjects with end-stage renal disease on hemodialysis. In: Presented at: Annual Meeting of the American College of Clinical Pharmacology; September 23-25, 2012; San Diego. Clin Pharmacol Drug Dev. 2012;1(4):175-229.
28. Upreti VV, Wang J, Barrett YC, et al. Effect of extremes of body weight on the pharmacokinetics, pharmacodynamics, safety and tolerability of apixaban in healthy subjects. Br J Clin Pharmacol. 2013;76(6):908-16.

29. Lassen MR, Raskob GE, Gallus A, et al. Apixaban or enoxaparin for thromboprophylaxis after knee replacement. N Engl J Med. 2009;361(6):594-604.

30. Pineo GF, Gallus AS, Raskob GE, et al. Apixaban after hip or knee arthroplasty versus enoxaparin: efficacy and safety in key clinical subgroups. J Thromb Haemost. 2013;11(3):444-51.

31. Lip GY, Andreotti F, Fauchier L, et al. Bleeding risk assessment and management in atrial fibrillation patients: a position document from the European Heart Rhythm Association, endorsed by the European Society of Cardiology Working Group on Thrombosis. Europace. 2011;13(5):723-46. 